\title{
A Novel Approach for Wind Speed Forecasting Using LSTM- ARIMA Deep Learning Models
}

\author{
Vikram Bali, JSS Academy of Technical Education, Noida, India \\ Ajay Kumar, JSS Academy of Technical Education, Noida, India \\ (iD https://orcid.org/0000-0002-3693-9701 \\ Satyam Gangwar, JSS Academy of Technical Education, Noida, India
}

\begin{abstract}
The term which is used to predict wind speed to produce wind power is wind speed forecasting. Deep learning, is a form of AI, basically indulging in artificial intelligence and thus can greatly increase the precision rate on larger datasets. In this research paper, the two techniques are being used together to obtain the better forecasting results. Both the techniques are forecasting based and combining LSTM and deep learning can increase the forecast rate because of the pattern remembering attribute of LSTM over a longer interval/period of time. If there is the inclusion of the ARIMA model the likelihood of a future value lying between two indicated limits is increased. So, overall if both the techniques are hybridized than it is most probable that the obtained results should be more accurate than both the techniques used separately. So, the main focus of this research article is on the efficiency and evaluation of hybridized LSTM-ARIMA model to predict wind speed forecasting.
\end{abstract}

\section{KEYWORDS}

Energy Usage, Load Prediction, LSTM Networks, Power Generation, Short Term Load Forecasting, Wind Energy, Wind Speed Forecasting

\section{INTRODUCTION}

Nowadays, the extensive use of non-renewable resources has become a major problem, as these resources are present in limited quantity and it's a need of time to save them for the future generation and certain measures must be taken for this purpose. Due to extensively availability of wind and low operating cost makes it the perfect natural resource that can utilize for the development of alternative source for the production of power so the better estimating will situate wind for better development and entrance into the worldwide vitality blend.

\section{Motivation Behind the Research Work}

The main motive of this research work is to find the alternative energy resources to meet up the need of time i.e. to save the energy resources for future generation. As we know that the natural resources 
are declining too fast, so as to save them, we have to take certain steps to use them for longer duration. Also maximum use of fossil fuels is polluting the environment, so the development of alternative energy resource is compulsory for the betterment of the earth but also development of these types of models in the modern era has become a basic need, as the traditional models are not quite up to the mark in terms of accuracy and costs.

\section{Importance of Wind Prediction}

As the energy demand is increasing quite rapidly and in contrary to it energy resources available at present is in less quantity, so as to overcome such problem, this type of alternative resources are used to kept consideration to meet the need of resource requirement. Since wind is renewable form of energy and is present everywhere, therefore can be utilized for the power production. The availability of wind is everywhere and free of cost which can be used as a proper tool to generate the power in useful manner (Wang, J. et al., 2017). Projecting wind speed is wind power estimation, and this is useful in energy epoch. And it is also performed to maintain the gap between energy usage and power generation. Also, the power generated from the wind energy is having low operating cost and thus help the users to use that power at a quite reasonable cost and thus results in providing power in the electricity prone area. This step can enhance the power production at a quite reasonable cost, and it will end up the balancing of the power production and power usage.

\section{Scope of Research Work}

According to (Liu et al., 2018), there are certain types of implications which can be observed by the wind projections such as diminished fees and fines for instabilities, Sustainable awareness superiority in the energy industry in live time or ' day ahead ', better effective design of projects, sustainment preparation (method to precise wind power projections which is valuable in lowering curtailment occurrence or duration to reduce cost of power generation), enhancing worker safety, and minimizing severe weather physiological impacts on wind power networks.

Numerous wind speed projections techniques have been evolved, but they have certain challenges with their reliability and consistency, as better the prediction model better will be the efficiency rate and thus results in the better utilization of generated power. This research work includes wind speed forecasting model for wind power generation so as to meet the present need of power requirement. (Cadenas et al., 2010) Several models were proposed for such type of predictions The models stated earlier have different constraints such as limited effectiveness, high operational expense, increased demand for capital and resources, strong variability and excessive requirement of training etc., so for solving some issues that are not fully resolved by the other models of prediction, the combined approach in deep learning with LSTM (Long Short Term Memory) and ARIMA (Autoregressive Integrated Moving-average) can lead to stronger wind related forecasts and which can help out in better power utilizations. The major scope of this research work is to develop a unique hybridized model comprising of LSTM-ARIMA, this model will have all the advance features as well as can sort out the various other problems which are being stated by the previously available models (Pourmousavi et al., 2013). The model discussed here uses the new techniques of machine learning and other useful aspects of projections and furthermore the obtained outcome or the projections are carried out to check the variations produced by wind due to its unpredictable nature. Whenever the predictions are produced, they are carried out in four distinct horizons, i.e. very-short duration type forecasting, short duration type forecasting, medium duration type forecasting, and long duration type forecasting. (Kavasseri et al., 2009The involvement of different parameters in deep learning models can also lead to better predictions of wind speed. Therefore, a distinctive wind speed forecast model needs to be developed with a more appropriate approach and with effective outcome. The hybrid model can be of excellent use in the forecasting of wind velocity.

The paper is divided into various sections like related work which basically includes all the four types of models i.e. very short duration type model, short duration type models, medium 
duration type models and lastly the long duration type models, here all the proposed models in their proper horizon distributions are described with their pros and cons, the next section comprises of proposed methodology which reveals research process that how the data is selected to the last step of result generation, in between these two process various other functions are being performed i.e. preprocessing, data visualization etc.. The main objective of this section is to discuss proposed LSTM-ARIMA algorithm and then the result section describes the comparison table with rmse values, prediction graphs and performance graphs of proposed methodology with existing algorithms. Finally, the last section describes the conclusion and future work of the current research work.

\section{RELATED WORK}

This section describes the various models proposed by various researchers in the current state of art. It includes the study of different models with different parameters and environment requirements. After the detailed study of literature review it is observed that the forecasting of wind speed can be done on the basis of four different horizons i.e. very short duration type of models ranging from few seconds only, short duration type models ranging from few $n$ minutes to 60 minutes, medium term duration type models ranging from a day ahead and long term forecasting ranging from one day to one week. Since a different method produces different outcomes in altering the atmosphere, circumstances, wind parameters, etc. so selection of good model with appropriate model is necessary.

\section{Very Short Duration Type Models}

This approach of wind speed forecasting has various models but one of model is a very short duration type model based on K-nearest neighbor classification (Yesilbudak et al., 2013). In this model, the error rate is high and the less precision is discovered. The model is quite independent on the sort of information set and also supports multiple tuppled inputs, but the main disadvantage of this model is that it shows inappropriate outcomes with fewer parameters. (Tronsco et al., 2015) develop a model on regression tree, it has the involvement of overall eight regression tree algorithms which derives out the small calculation time and thus help out in the outcome can be readily viewed in the regression tree. But it is not possible to approximate the same function and also Global models have small computing costs compared to local models. (Pourmousavi et al., 2013) proposed another paper with an advance model using ANN model so as to figure out the reduction in uncertainty while error estimation and also reduction in time of calculation but here excessive training data can be clearly observed which is quite tough to handle. (Jiang et al., 2013) suggested a model based on Bayesian structure, the main advantage of this model is that it gives out the number of results in the form of different sets so a variety of results can be obtained. The main disadvantages of this model are lower efficiency, more faults and higher interruptions due to its complex structure. (Gupta et al., 2017) proposed the various different methodologies for document clustering using K-nearest neighbor and ANN. (Gupta et al., 2017) had also gone through the numerous semantic resemblances analysis techniques utilizing latent semantic analysis.

\section{Short Duration Type Models}

The models based on this approach are discussed here such as a methodology based on hybridizing of WTT (Wavelet Transform Technique) + SAM (Seasonal Adjustment Method) + RBFN (Radial Basis Function Neural Network) (Zhang et al., 2013). This model shows lower error rate and good performance rate due to hybridization of various techniques, but this also requires previous knowledge of various technologies. The model based on ANN provides gaining expertise from training data, demonstrates robustness and high tolerance of errors but the main disadvantage of this strategy is that it requires large training statistics sets and thorough analysis is required due to its flexible structure results in inconsistent outcomes (Cadenas et al., 2009). (Damousis et al., 2004) suggested a fuzzy approach which is used where systems are quite hard to model and tackle and is comparatively less 
complicated than other models, but the performance rate reduces where the terrain is complicated and training of data for such models requires quite longer durations of time. (li et al., 2011) proposed a Bayesian adaptive $-\mathrm{NN}$ model which provides $\mathrm{NN}$ with a reliable, adaptive and relatively precise consequence but this type of model does produce good results for forecasting of outcomes for one hour ahead type predictions in comparison several other models. (Liu et al., 2014) gave a hybridized model consisting of WT+SVM+GA (Genetic Algorithms) shows stability in model and also it controls or we can say it eliminates the variances in the wind by using wavelet transforms (WT). But the major disadvantages of this model are that it uses only one parameter at a moment and high data analysis is absent. (Liu et al., 2018) suggested a Smart deep learning model which shows Reliable and efficient model with estimate improved results for variations. But this deep learning-based model requires more computing resources to be work on. (Kumar et al., 2018) stated the use of Rock Predication optimization approach using ANN. (Kumar et al., 2018) mentioned different methods Using ANN and ML for electricity loading. (Jha et al., 2012) advised the use of compression algorithms using the technique of goal learning. (Jha \& Bali, 2012) mentioned the use of compression techniques using a programming strategy. The model comprising of SC+SVM+ANN (Wang et al., 2017) shows the functionality to keep up a steady power supply framework that considers the stochastic idea of wind speed, exact breeze speed determining is critical here a mixed estimating model is built up, the SCS (Similar Coefficient Sum) technique is used to process the first wind speed information, and the SVM model whose parameters are tuned by a man-made brainpower model is worked to make conjecture (Wang \& Wang, 2017). (Huang et al., 2018) has discussed the estimation of evolved results from various calculation was contrasted with that of four other computerized reasoning calculations normally utilized in wind speed determining and also various other methods were also compared while testing of obtained values in terms of error rates (Huang et al., 2018).

\section{Medium Duration Type Models}

The models proposed in this type of approach are discussed here i.e. (Cadenas et al., 2010) suggested the ARIMA-ANN model which shows Strong precision in wind speed and is used in linear and nonlinear time sequence. The disadvantage of this model is that model shows high precision rates when used independently. (Wang et al., 2015) proposed a Hybrid model which shows high generalization performance, but this model has few disadvantages too i.e. Computation is very challenging and takes a bit longer training time for the training of data items. (Filik \& Filik, 2017) mentioned the approach which uses ANN + Metrological based equations with various parameters, here an information observing framework are utilized which can delicately measures in milliseconds time interim and records the estimations of climate temperature, wind speed, wind course and climate pressure and the results can be rehashed for longer periods. Utilizing various hybrid models of different channels, it tends to be explored on the impacts of wind speed expectation (Filik et al., 2017). (Zhang et al., 2019) uses EMDBP on the Lorentz system, it was to utilized genuine breeze speed information to take a recreation test and investigated the improvement impact of the primer anticipating succession of wind speed affected by Lorenz condition in the transient turmoil and confusion here the improved model debilitated the irregular vacillation of wind speed (Zhang et al., 2019).

\section{Long Duration Type Models}

In this section, various long duration type models are being discussed. The main purpose of such types of model is to optimize the cost and in other operational management activities. i.e. (Kavasseri et al., 2009) proposed an f-ARIMA model which is basically beneficial in modeling time series data and the design is very fundamental type, but the major demerit of this model is that non-linear framing of problems is troublesome and lot of information requirements is necessary to produce good results. (Barbounis \& Theocharis, 2006) suggested the local recurrence NN model which offers a stable design and various types of wind variations are erased by WT. But the model is applicable of using only just one parameter at a moment and also comprehensive quantitative analysis is missing in this model. 
(Barbounis et al., 2006) proposed the local recurrent NN model which Offers good sustained learning stability and it requires smaller computational as well as smaller storage. But this model considered being a complicated structure one as it has several parameters to work with. (Mishra \& Das, 2017) suggested the use of kernel based neural network and a precise short-term wind speed model based on the powerful variants of the kernel ridge pseudo inverted neural network (KRPINN). Since the individual piece capacities based KRPINN will not deliver precise estimates under tumultuously fluctuating breeze speed conditions, a straight blend of individual portion capacities is utilized to manufacture the multi bit edge pseudo backwards neural system.

\section{Summary and Research Gaps}

In all the above models, various limitations such as low precision rate require datasets of multiple tuples. It requires a variety of parameters to show accurate results and the maintenance cost is quite high as compared to others. Also, these models deal with greater number of training sets with numerous types of interruptions during the functionality of models. During the formation of such models the physical assessment of models is also mandatory so as to generate the better results with higher accuracy rates.

Due to constant modifications in wind velocity, some wind velocity forecast designs suggested to satisfy the need for energy generation, but the suggested designs show bad precision rates, low effectiveness and other constraints that can abrupt the continuous power generation.

So, our proposed model using two techniques i.e. LSTM-ARMA resolve all the above-mentioned limitations and thus provide us with the quite effective and robust projection model with higher accuracy rate. The proposed methodology is discussed in the next section.

\section{PROPOSED METHODOLOGY}

Through extensive literature survey, we can conclude that the models developed in the field of prediction of wind is not quite up to the mark there is a need to generate a new model in order to resolve the limitations of existing models. So, the proposed methodology for wind speed predictions uses the deep learning approaches to improve the accuracy of the existing models (Kavasseri et al., 2009). It uses LSTM-ARIMA model networks which can be helpful in generating more accurate results and better efficiency. LSTM is quite alike RNN i.e. RNNs are commonly recognized as a technique that offers an easy manner to address complicated and undefined issues. (Pourmousavi et al., 2013), has described that RNN has several benefits, but one of the most known of these is that it can effectively benefit from watching information sets. Here the back propagation is through time as the output gradient depends on the previous values other than the current values only.

This section constitutes of the various stages like analysis of raw data, pre-processing of data, information prediction using deep learning approach i.e. LSTM-ARIMA. In order to create a more reliable, effective and precise model for forecasting wind speed, all the steps listed in the suggested methodologies must be followed correctly. In sequence, first of all, the raw data is collected and carefully monitored to check the cleaning of data is required or not. i.e. the available data is ready to be used without any changes, unless certain pre-processing techniques are used over the available dataset to make it relevant to use. The data set available here has many parameters, but our proposed approach requires only few parameters or attributes, so the preprocessing is done in which the dimensionality of the data is reduced. The information thus acquired in this step is refined data. Now, the proposed algorithm can be used on this data to create our own model to predict wind speed outcomes. In the last step the accuracy of proposed algorithm is evaluated in the form of distinct types of percentage errors and compares it with existing algorithms. The workflow in the proposed methodology based on LSTM and ARIMA for wind speed forecasting is given in Figure 1.

The elements such as raw data, data visualization/ preprocessing, data prediction, visualization is described as follows. 


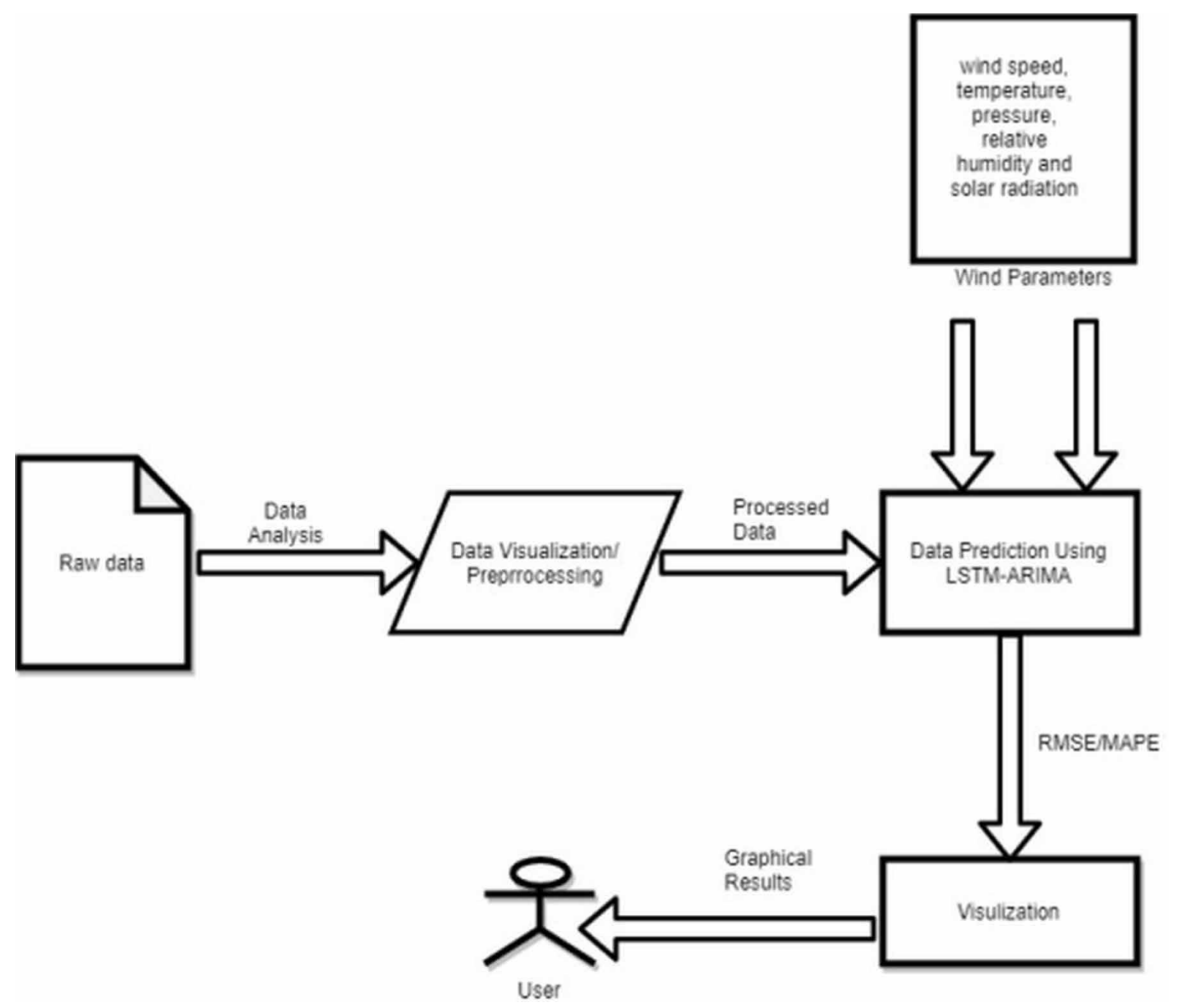

\section{Raw Data (Data Analysis)}

Basically, the raw data is the unprocessed or the data that is not ready to be used for any extraction of information. It is also regarded as the source information that was not passed through any processing technique either manually or by any algorithm or automated machine. Basically, here data analysis is done so as to select the relevant data of our use. The primary data set is taken from the data sets of kaggle. The accessible dataset has tupples (420552) with attributes (15). The input data set is in second's format, so the next stage is to process data and use the algorithms and procedures to make data efficient and healthy to use. The Attributes used in the dataset is given in Figure 2.

\section{Preprocessing of Data}

The primary goal in the preprocessing stage is to select the necessary information including the following characteristics: wind velocity, temperature, pressure, relative humidity and solar radiation. Preprocessing here is accomplished through visualization method and training is achieved through the recurring neural network, it is really a must-follow step in any proposed methodology to develop a distinctive and more accurate model. Data processing is essentially the filtering of data in order to make it easier to use. After the dataset is trained with the inclusion of the following characteristics is shown in Figure 3. In these proposed methodologies after the dataset is trained with the inclusion of following attributes is depicted below. Keras is minimized, simple to adapt, abnormal state Python 
Figure 2. Dataset (adapted from [https://www.kaggle.com/pankrzysiu/weather-archive-jena])

\begin{tabular}{|c|c|c|c|c|c|c|c|c|c|c|c|c|c|c|c|}
\hline$\Delta$ & A & B & c & D & $E$ & $\mathrm{~F}$ & G & H & 1 & J & K & L & M & $\mathrm{N}$ & 0 \\
\hline 1 & Date Time & $p$ (mbar) & $\mathrm{T}(\operatorname{deg} C)$ & Tpot (K) & \multicolumn{2}{|c|}{ Tdew (dęrh (\%) } & \multicolumn{4}{|c|}{ VPmax (m VPact (mb VPdef (ml sh $(\mathrm{g} / \mathrm{kg})$} & \multicolumn{3}{|c|}{$\mathrm{H} 2 \mathrm{OC}\left(\mathrm{mn}\right.$ rho $\left(\mathrm{g} / \mathrm{m}^{*} \mathrm{wv}(\mathrm{m} / \mathrm{s})\right.$} & \multicolumn{2}{|c|}{ max. wv (I wd (deg) } \\
\hline 2 & $01.01 .200 \leqq$ & 996.52 & -8.02 & 265.4 & -8.9 & 93.3 & 3.33 & 3.11 & 0.22 & 1.94 & 3.12 & 1307.75 & 1.03 & 1.75 & 152.3 \\
\hline 3 & $01.01 .200 \varsigma$ & 996.57 & -8.41 & 265.01 & -9.28 & 93.4 & 3.23 & 3.02 & 0.21 & 1.89 & 3.03 & 1309.8 & 0.72 & 1.5 & 136.1 \\
\hline 4 & 01.01 .2005 & 996.53 & -8.51 & 264.91 & -9.31 & 93.9 & 3.21 & 3.01 & 0.2 & 1.88 & 3.02 & 1310.24 & 0.19 & 0.63 & 171.6 \\
\hline 5 & $01.01 .200 \mathrm{~s}$ & 996.51 & -8.31 & 265.12 & -9.07 & 94.2 & 3.26 & 3.07 & 0.19 & 1.92 & 3.08 & 1309.19 & 0.34 & 0.5 & 198 \\
\hline 6 & 01.01 .2005 & 996.51 & -8.27 & 265.15 & -9.04 & 94.1 & 3.27 & 3.08 & 0.19 & 1.92 & 3.09 & 1309 & 0.32 & 0.63 & 214.3 \\
\hline 7 & $01.01 .200 \varsigma$ & 996.5 & -8.05 & 265.38 & -8.78 & 94.4 & 3.33 & 3.14 & 0.19 & 1.96 & 3.15 & 1307.86 & 0.21 & 0.63 & 192.7 \\
\hline 8 & 01.01 .200 ؛ & 996.5 & -7.62 & 265.81 & -8.3 & 94.8 & 3.44 & 3.26 & 0.18 & 2.04 & 3.27 & 1305.68 & 0.18 & 0.63 & 166.5 \\
\hline 9 & 01.01 .200 ؛ & 996.5 & -7.62 & 265.81 & -8.36 & 94.4 & 3.44 & 3.25 & 0.19 & 2.03 & 3.26 & 1305.69 & 0.19 & 0.5 & 118.6 \\
\hline 10 & $01.01 .200 \mathrm{~s}$ & 996.5 & -7.91 & 265.52 & -8.73 & 93.8 & 3.36 & 3.15 & 0.21 & 1.97 & 3.16 & 1307.17 & 0.28 & 0.75 & 188.5 \\
\hline 11 & $01.01 .200 \leqslant$ & 996.53 & -8.43 & 264.99 & -9.34 & 93.1 & 3.23 & 3 & 0.22 & 1.88 & 3.02 & 1309.85 & 0.59 & 0.88 & 185 \\
\hline 12 & $01.01 .200 \leqslant$ & 996.62 & -8.76 & 264.66 & -9.66 & 93.1 & 3.14 & 2.93 & 0.22 & 1.83 & 2.94 & 1311.64 & 0.45 & 0.88 & 183.2 \\
\hline 13 & 01.01 .200 s & 996.62 & -8.88 & 264.54 & -9.77 & 93.2 & 3.12 & 2.9 & 0.21 & 1.81 & 2.91 & 1312.25 & 0.25 & 0.63 & 190.3 \\
\hline 14 & $01.01 .200 \subseteq$ & 996.63 & -8.85 & 264.57 & -9.7 & 93.5 & 3.12 & 2.92 & 0.2 & 1.82 & 2.93 & 1312.11 & 0.16 & 0.5 & 158.3 \\
\hline 15 & 01.01 .2005 & 996.74 & -8.83 & 264.58 & -9.68 & 93.5 & 3.13 & 2.92 & 0.2 & 1.83 & 2.93 & 1312.15 & 0.36 & 0.63 & 184.8 \\
\hline 16 & 01.01 .200 s & 996.81 & -8.66 & 264.74 & -9.46 & 93.9 & 3.17 & 2.98 & 0.19 & 1.86 & 2.99 & 1311.37 & 0.33 & 0.75 & 155.9 \\
\hline 17 & 01.01 .200 S & 996.81 & -8.66 & 264.74 & -9.5 & 93.6 & 3.17 & 2.97 & 0.2 & 1.85 & 2.98 & 1311.38 & 0.07 & 0.5 & 272.4 \\
\hline 18 & $01.01 .200 \leqslant$ & 996.86 & -8.7 & 264.7 & -9.55 & 93.5 & 3.16 & 2.95 & 0.21 & 1.85 & 2.96 & 1311.64 & 0.32 & 0.63 & 219.2 \\
\hline 19 & 01.01 .200 s & 996.84 & -8.81 & 264.59 & -9.66 & 93.5 & 3.13 & 2.93 & 0.2 & 1.83 & 2.94 & 1312.18 & 0.18 & 0.63 & 167.2 \\
\hline 20 & 01.01 .200 s & 996.87 & -8.84 & 264.56 & -9.69 & 93.5 & 3.13 & 2.92 & 0.2 & 1.83 & 2.93 & 1312.37 & 0.07 & 0.25 & 129.3 \\
\hline 21 & 01.01 .200 s & 996.97 & -8.94 & 264.45 & -9.82 & 93.3 & 3.1 & 2.89 & 0.21 & 1.81 & 2.9 & 1313.01 & 0.1 & 0.63 & 115.3 \\
\hline 22 & 01.01 .200 s & 997.08 & -8.94 & 264.44 & -9.8 & 93.4 & 3.1 & 2.9 & 0.2 & 1.81 & 2.9 & 1313.15 & 0.3 & 0.75 & 149.3 \\
\hline 23 & $01.01 .200 \subseteq$ & 997.1 & -8.86 & 264.52 & -9.76 & 93.1 & 3.12 & 2.9 & 0.22 & 1.81 & 2.91 & 1312.78 & 0.29 & 0.75 & 149.7 \\
\hline 24 & 01.01 .200 S & 997.06 & -8.99 & 264.39 & -9.99 & 92.4 & 3.09 & 2.85 & 0.23 & 1.78 & 2.86 & 1313.39 & 0.12 & 0.63 & 231.7 \\
\hline 25 & 01.01 .200 s & 996.99 & -9.05 & 264.34 & -10.02 & 92.6 & 3.07 & 2.85 & 0.23 & 1.78 & 2.85 & 1313.61 & 0.1 & 0.38 & 240 \\
\hline
\end{tabular}

Figure 3. Processed dataset

\begin{tabular}{|c|c|c|c|c|c|}
\hline B & $c$ & D & E & $\mathbf{F}$ & G \\
\hline \multicolumn{2}{|c|}{ Wind Sper Pressure } & \multicolumn{2}{|c|}{ Relative H Temperat } & \multicolumn{2}{|c|}{ Solar Radiation } \\
\hline 3.011042 & 986.7618 & 72.29586 & 13.52266 & o & \\
\hline 3.091243 & 986.4414 & 75.37619 & 12.83581 & o & \\
\hline 3.007649 & 985.7365 & 78.4052 & 12.19806 & 0 & \\
\hline 2.926715 & 985.5257 & 81.04298 & 11.5835 & $\mathbf{0}$ & \\
\hline 2.915177 & 985.6619 & 82.54851 & 11.02958 & o & \\
\hline 1.937899 & 985.7232 & 65.27849 & 8.098655 & $\mathbf{0}$ & \\
\hline 1.815175 & 986.2491 & 67.09318 & 7.806705 & $\mathbf{0}$ & \\
\hline 1.61531 & 987.0389 & 64.03548 & 9.036408 & o & \\
\hline 1.439088 & 987.8974 & 55.10566 & 12.15828 & 159 & \\
\hline 1.063374 & 988.2119 & 47.16694 & 14.9653 & 363 & \\
\hline 0.545695 & 988.0545 & 36.74709 & 18.95608 & 533 & \\
\hline 0.980117 & 987.4639 & 29.15702 & 21.35405 & 649 & \\
\hline 1.411915 & 986.3862 & 27.59307 & 22.16126 & 698 & \\
\hline 1. 747909 & 985.7365 & 27.2747 & 22.25622 & 675 & \\
\hline 2.005287 & 985.5215 & 27.81929 & 21.72588 & 582 & \\
\hline 2.206879 & 985.2797 & 30.02328 & 20.50727 & 430 & \\
\hline
\end{tabular}

library keep running over Tensor Flow system. It is made with focal point of seeing profound learning methods, for example, making layers for neural systems keeping up the ideas of shapes and numerical.

\section{Data Predictions Using LSTM-ARIMA Model}

Predictions are produced through the execution of appropriate algorithms in this stage. In the following paper, the two algorithms are combined to predict results, i.e. LSTM (long-term memory) and others are ARIMA (Auto Regressive Integrated Moving Average). Both algorithms represent distinct forecast. And the outcome was acquired in the form of RMSE and MAPE values. And then these predictions are further used for visualization of data.

LSTM can handle multiple effective issues; it is nothing but a neural network that works in the field of deep learning. Since LSTM has the most beneficial assets of tracing past events for a quite long 
period of time, it provides wider aspects in the forecasting models. Because of this property, multiple methodologies are used to draw out certain kinds of projections. Most importantly, it displays RNN's property, so it loops back to hidden layers to make the results / outputs more precise that can be used to produce effective models. LSTM uses different functions in calculation of hidden states. LSTM contains a memory cell, having four main elements including an input gate, a neuron, a forget gate and one more a output gate. Interaction between environment and memory cells is achieved by the involvement of LSTM gate only. There is a need to propagate two steps back to calculate a gradient at three and sum the gradient value. Working of LSTM gate layer interaction is shown in Figure 4.

In LSTM process the output of one node is given as a input to the another state. The updating of new state takes place in the form given in Equation 1:

Figure 4. Working of LSTM gates and layer interactions

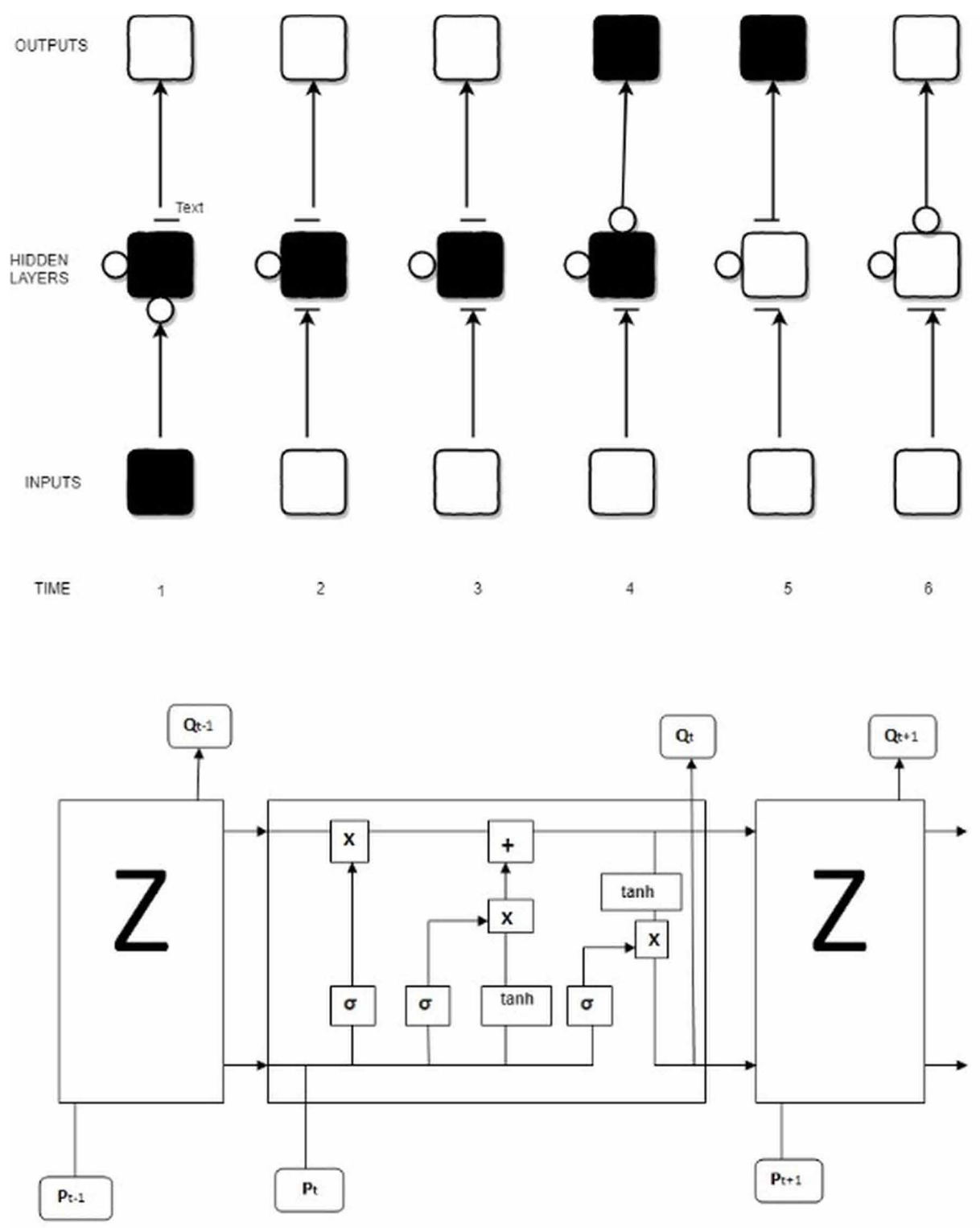


$B v=f v^{*} B v-1+i v^{*} B v^{\prime}$

where:

Bv: new state or updated state

$f v$ : function value

$\mathrm{Bv}-1$ : old state

$i$ v: input value

Bv': candidate value

In case of ARIMA model ARIMA stands for "auto regressive integrated moving average," these models worked through data linked to the time series. For the purpose of time series data analysis and forecasting, an ARIMA model is a category of demographic systems. In this way, it can also be examined i.e.:

- AR (Auto Regressive): It is implied on those types of models were a clear view of dependent relationship can be observed between the observations and the observations which are lagging of certain resources or the information;

- I (Integrated): Subtraction of findings/readings occurs here, i.e. differentiation in findings/ readings at two stages (past and present one), so that findings can be obtained further;

- MA (Moving Average): It is nothing but just to observe the reliability over the observations and the remaining errors from a moving average model used over the lagging observations.

ARIMA notations can be seen below:

[AR by 1]

$\left[\begin{array}{lll}I & b y & f\end{array}\right]$

$[$ MA by $\mathrm{m}]$

Most important things which are kept in mind while predicting using ARIMA model is that of ensuring stationary of value of ' $\mathrm{f}$ ' and then find the values for ' $\mathrm{l}$ ' and ' $\mathrm{n}$ ' using techniques such as unit test roots, ACF (Autocorrelation function) and PACF (Partial Autocorrelation function) and then estimate the remaining values and select the appropriate one out of these.

To build a time series model issuing ARIMA, we need to study the time series and identify $1, \mathrm{f}, \mathrm{n}$ :

- Ensuring Stationarity: Determine the relevant values of f;

- Identification: Use ACF, PACF and unit root trials to determine the best and selective values of $1 \& \mathrm{n}$ and here 1 is the AR order, $\mathrm{f}$ is the integration order, $\mathrm{n}$ is the MA order;

- Estimation: Estimate an ARIMA model using 1, f, \& n values you believe are suitable or not;

- Diagnostic checking: Check the remainder of measured ARIMA models to see if they are white noise; choose the finest model with well-behaved remaining ones;

- Forecasting: For in-sample forecasting, produce from the sample forecasts or the set aside the last few data points.

The model proposed in neural network here uses LSTM-ARIMA to forecast wind speed for predicting purpose. The requirement of data is less and the result is accurate. The step wise description of proposed hybrid LSTM-ARIMA algorithm is given as below in the next section. 


\section{Hybrid LSTM+ ARIMA Algorithm}

Step 1: Data sets [train, dev]

Step 2: Read the files [train_csv/X/Y, test_X/Y]

Step 3: Now train the CSV file i.e. train_csv

Step 4: After the training of the file continue to define the model

Model=sequential

Model.add LSTM (units= 100)

Model.add Dense (1), activation= "double-tanh"

Model.add Flatten

Step 5: Repeat the process

Step 6: Now do the forward_propagation model with train_X

Step 7: Now do the backward_propagation model with train_Y

Step 8: Refurnished or updation of parameters of model

Step 9: In all Time series Ti in data do,

Model $=$ NULL

Step 10: For all order in orders do,

Rorder $=$ For ARIMA [Ti, order $]$

Step 11: Add Rorder to models

Step 12: unused $=X-$ predict $(\mathrm{Ti}, \mathrm{Rfit})$

Step 13: Add unused to $X$

Step 14: Add unused to $Y$

Step 15: Reserve/ save $X$ and $Y$

Step 16: Now train_RMSE, train_MAPE = model (train_X, train_Y)

And dev_RMSE, dev_MAPE $=$ model $($ dev_X, dev_Y)

Step 17: End repeat

Step 18: Test_RMSE, test_MAPE $=$ model (test_X, test_Y)

Step 19: END

\section{Visualization}

Visualization is the method of evaluating the outcome acquired, in this stage the expected wind data is visualized and the outcome received in the form of RMSE and MAPE is correctly evaluated to produce the expected graphs and other needed visual representations.

\section{RESULTS}

The primary focus of this section is to explore the different outcomes produced during the work of the suggested methodology. In this paper, the novel approach LSTM-ARIMA is used with the dataset containing 420528 tuples having data attributes in terms of seconds and after preprocessing it is converted into hourly basis and reduced to the dataset containing 1751 tuples. RSME value is used to show the predicted result. Table 1 shows the performance rate of LSTM and LSTM-ARIMA hybrid model i.e. RMSE value comparison.

The predicted result was quite different in terms RMSE value. Here MSE value is calculated by this formula given in Equation 2 and RMSE value is calculated by using the formula given in Equation 3:

$$
M S E_{c}=\frac{1}{M}\left(\sum_{r=1}^{M} \mathrm{e}_{r+c}^{2}\right)
$$


Table 1 Models with their RMSE comparison value

\begin{tabular}{|l|l|l|}
\hline \multicolumn{1}{|c|}{ S.NO. } & \multicolumn{1}{|c|}{ MODEL } & \multicolumn{1}{c|}{ RMSE Value } \\
\hline 1 & LSTM & 0.427 \\
\hline 2 & LSTM-ARIMA & 0.254 \\
\hline 2 & SVM & 0.768 \\
\hline
\end{tabular}

$$
R M S E_{C}=\sqrt{M S E_{c}}
$$

where:

e: predicting error

M: total no. of observations

Thus, the accuracy rate of LSTM-ARIMA model is higher than the LSTM and SVM one:

\section{SVM $<$ LSTM $<$ LSTM-ARIMA}

This section has various graphs, comparison graphs which were generated during the proposed methodologies. The parameters that were taken under consideration while the implementation of both the methodologies it includes (wind speed, pressure, relative humidity, temperature, solar radiation). The visualized parameters are those parameters which are extracted from the whole lot of dataset and used for the implementation process. The selection of different variables is necessary to develop the good model as using of all the variables might show inappropriate behavior in terms of projections. The visualized or the parameter used for prediction is shown in Figure 5 where $x$-axis represents the time stamp and y-axis represents the number of units.

Figure 5. Visualized parameters

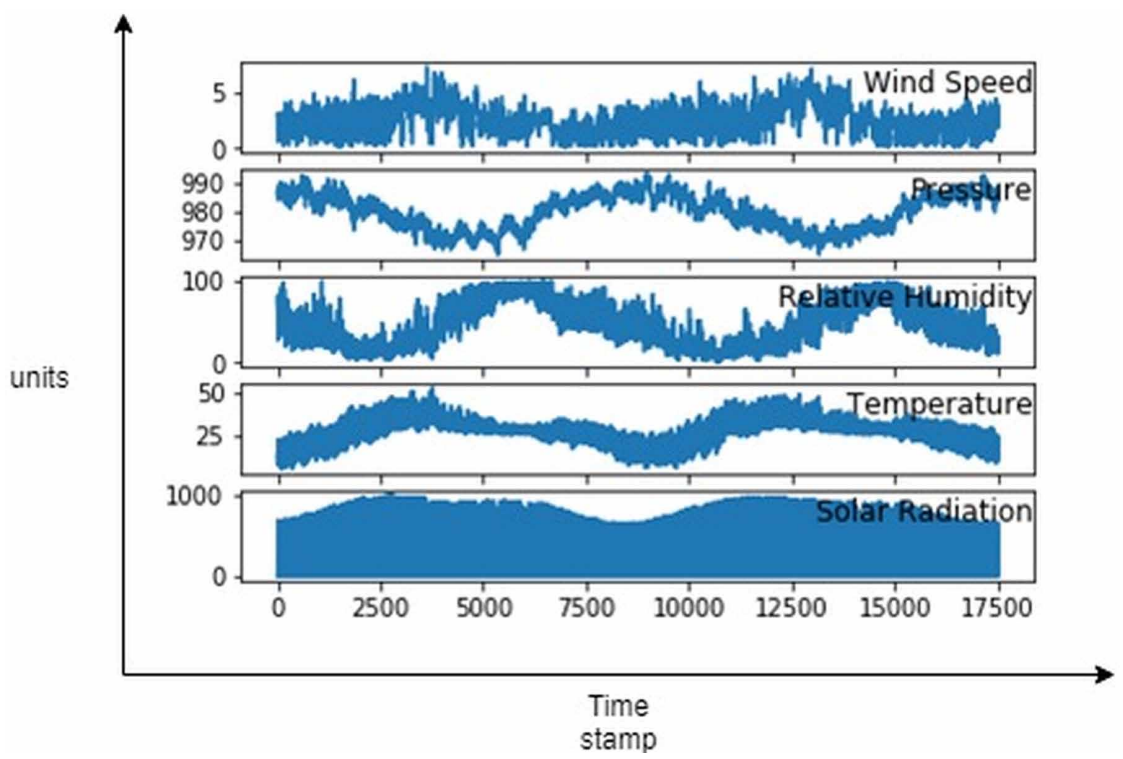


In Figure 6, the graph demonstrates the training and testing loss, while the data is being trained for methodology execution. The graph below demonstrates the real wind speed and forecast one for proposed LSTM-ARIMA methodology. Some kinds of loss happened during data training and testing, which is due to the irregular occurrence of information. These losses are also caused by the failures in the algorithms.

Figure 6. Training and testing Losses

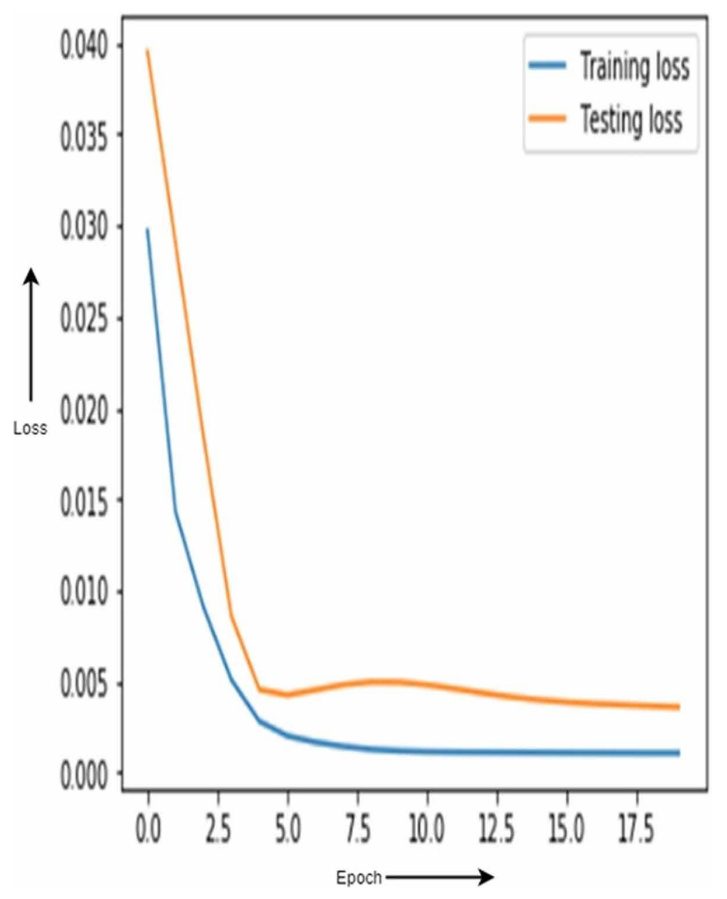

The Figure 7 depicts the graph which shows the variations in wind speed when it is been tested for the trained data sets. As it is clearly seen that the generated graph shows less variations in predictions .so it is must that it will provide the better results that the other models. Here real speed of wind is given by blue color and in contrary to it the speed wind which is predicted is in red color.

Finally, the result obtained by the LSTM-ARIMA model in terms of RMSE value is 0.254 , which is quite better and efficient result in terms of predictions. According to the obtained result which being generated using RMSE value of each model i.e. LSTM, SVM, LSTM-ARIMA. After this it is clearly observe that which model has the best accuracy rate. By analyzing we conclude that hybridization of LSTM-ARIMA is better than LSTM alone itself.

\section{Performance Analysis}

This section describes the performance of our proposed algorithm with the existing algorithms. The performance basis graphs are generated by using RMSE value of all the models i.e. SVM, LSTM and LSTM-ARIMA. As it can be observed clearly from the graph that RMSE value for LSTM is less as compared to the SVM. The error rate in LSTM is 0.427 while in case of SVM it is 0.768 , it depicts that LSTM model is more efficient than SVM model. The performance graph of SVM with LSTM is shown in Figure 8. 


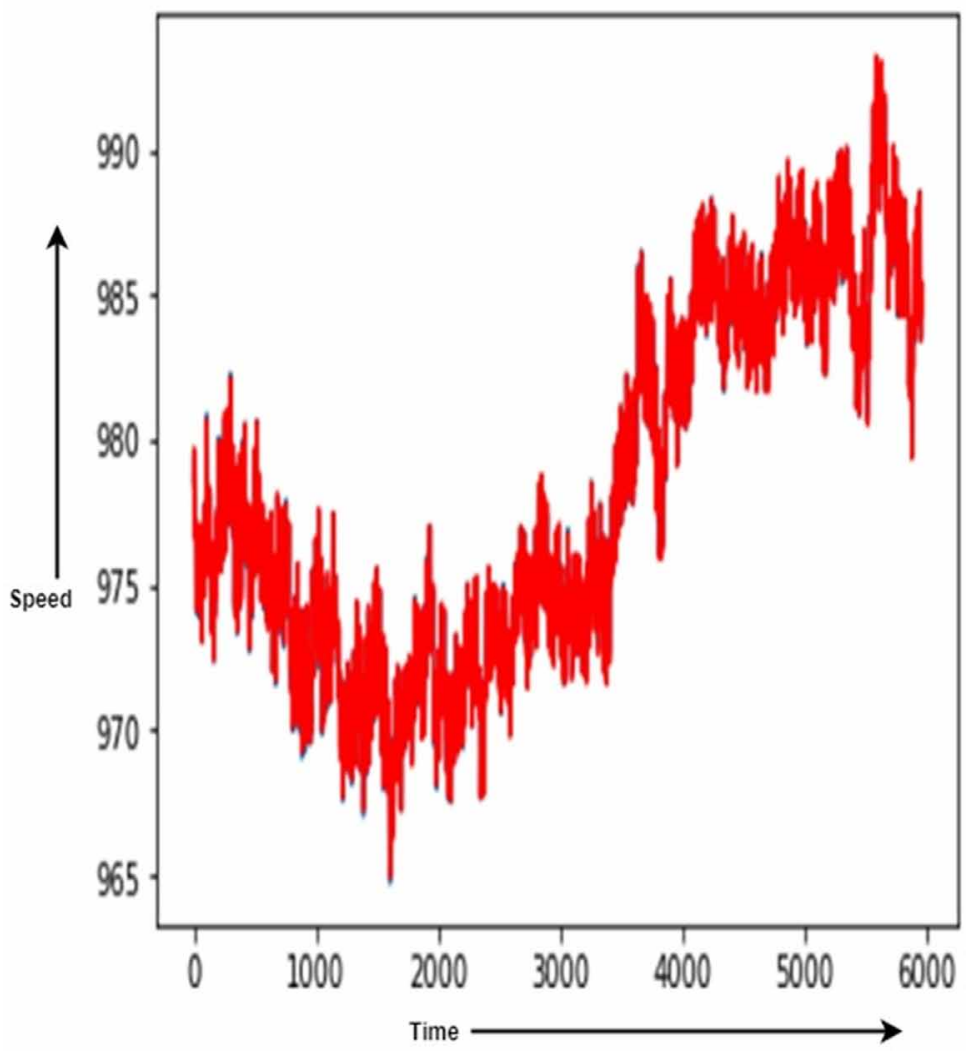

Similarly, it can be observed that RMSE value for LSTM-ARIMA is less as compared to the LSTM. The error rate in LSTM-ARIMA is 0.254 while in case of SVM it is 0.427 , it depicts that LSTM model is more efficient than SVM model. The performance graph of LSTM-ARIMA with LSTM is shown in Figure 9.

\section{CONCLUSION}

After the detailed observation of the results LSTM-ARIMA turns out to be quite effective technique as the error rate in LSTM-ARIMA is quite less so it can be used more frequently in the forecasting techniques as compared to another one. As we know that LSTM with deep learning can be implemented so as to obtain the more efficient result in the forecasting approaches because of its pattern recognition property for a long duration of time or we can say that if LSTM is hybridized with other models to generate more accurate models with efficient prediction. It can analyze huge information efficiently. It has the benefit of generalization and the capacity to approximate high dimensional function. In case of wind speed predictions, it can be used to maintain the gap between power generation and power utilization. The Long- Short Term Memory model is therefore shown to be sophisticated and practical in the forecast of wind power. Similar case is here when the LSTM is hybridized with the ARIMA model it gains the features of both the techniques and the results being obtained shows the best accuracy rate as comparison to other models. As much as better the prediction rate of wind speed it can be used to deduce the gap between energy production and energy consumption and other functionalities linked to wind. But while observing the model it can also be concluded that certain 
International Journal of Agricultural and Environmental Information Systems

Volume $11 \cdot$ Issue $3 \cdot$ July-September 2020

Figure 8. Performance graph of SVM and LSTM

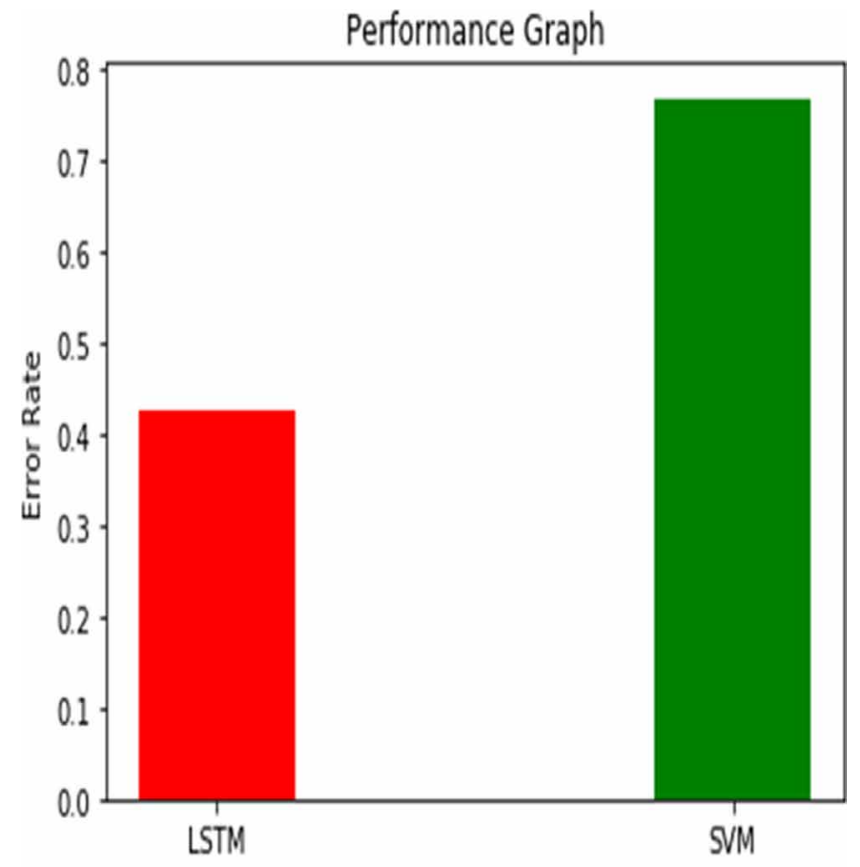

Algorithm

Figure 9. Performance graph of LSTM-ARIMA and LSTM

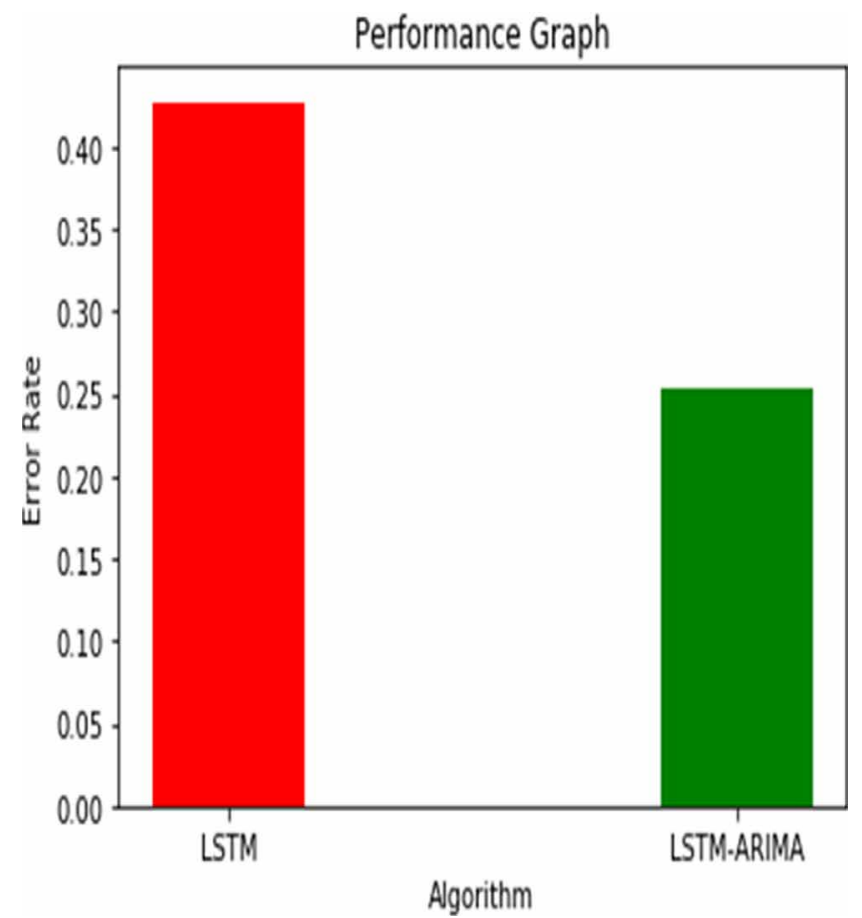


types of issues are must be kept in mind so as to enrich the model efficiency i.e. due to variable nature of wind speed it is essential to choose suitable input variables to construct an effective model of forecasting. Also, for distinct designs, distinct variables are required i.e. A physical model, it utilizes physical factors to forecast potential wind velocity and direction, so the input factors will be physical or meteorological data and the most specifically Selection of time duration for which the prediction is to done and then the selection of an appropriate model. Finally, combining models to make a hybrid model is quite a cumbersome task and must be done carefully.

\section{FUTURE SCOPE}

The main objective of this work is to develop a unique model to provide better wind speed prophecy. It has been concluded in the previous section that our proposed algorithm provides better results in comparison of other existing models. So, in order to improve the accuracy of the model, it is observed that one or more techniques can be used together. Also, more parameters as well as larger data sets may be applied so to develop more efficient as well more decent and accurate prophecy model. This type of models may be further elaborated to be used for other time durations also so that it might be possible that other method involvement may prove the less costing but high-outputs. 


\section{REFERENCES}

Barbounis, T. G., Theocharis, J. B., Alexiadis, M. C., \& Dokopoulos, P. S. (2006). Long-term wind speed and power forecasting using local recurrent neural network models. IEEE Transactions on Energy Conversion, 21(1), 273-284. doi:10.1109/TEC.2005.847954

Barbounis, T. G., \& Theocharis, J. B. (2006). Locally recurrent neural networks for long term wind speed and power predictions. Neurocomputing, 69(4-6), 466-496. doi:10.1016/j.neucom.2005.02.003

Cadenas, E., Rivera, W., Campos-Amezcua, R., \& Herd, C. (2016). Wind Speed prediction using a univariate ARIMA model and a Multivariate NARX Model. Energies, 109(9), 2-15.

Cadenas, E., \& Rivera, W. (2009). Short term wind speed forecasting in La Venta, Oaxaca, Mexico, using artificial neural networks. Renewable Energy, 34(1), 274-278. doi:10.1016/j.renene.2008.03.014

Cadenas, E., \& Rivera, W. (2010). Wind speed forecasting in three different regions of Mexico, using a hybrid ARIMA-ANN model. Renewable Energy, 35(12), 2732-2738. doi:10.1016/j.renene.2010.04.022

Chenhong, Z., Penghui, W., \& Yuanhang, Z. (2017). Wind Speed Prediction Research Based on Time Series Model with Residual Correction. In Proceedings of the 2nd International Conference on Power and Renewable Energy (pp. 466-470). Academic Press. doi:10.1109/ICPRE.2017.8390579

Damousis, G. I., Alexiadis, M. C., Theocharis, J. B., \& Dokopoulos, P. S. (2004). A Fuzzy model for wind speed prediction and power generation in wind parks using spatial correlation. IEEE Transactions on Energy Conversion, 19(2), 352-361. doi:10.1109/TEC.2003.821865

Filik, U., \& Filik, T. (2017). Wind Speed Prediction Using Artificial Neural Networks Based on Multiple Local Measurements in Eskisehir. In Proceedings of the 3rd International Conference on Energy and Environment Research (pp. 264-269). Academic Press. doi:10.1016/j.egypro.2016.12.147

Gupta, A., Gautam, J., \& Kumar, A. (2017). A Survey on the methodologies used for semantic document clustering. In Proceedings of the IEEE International Conference on Energy, Communication, Data Analytics and Soft Computing (pp. 671-675). IEEE Press. doi:10.1109/ICECDS.2017.8389521

Gupta, A., Kumar, A., \& Gautam, J. (2017). A survey on semantic similarity measures. International Journal for Innovative Research in Science \& Technology, 3(12), 243-247.

Huang, C., \& Kuo, P. (2018). A Short-Term Wind Speed Forecasting Model by Using Artificial Neural Networks with Stochastic Optimization for Renewable Energy Systems. Energies, 11(10), 1-20. doi:10.3390/en11102777

Jha, P. C., \& Bali, V. (2012). Goal programming approach for selection of COTS components in designing a fault tolerant modular software system under consensus recovery block scheme. International Journal of Computer and Communication Technology, 3(1), 1-8.

Jha, P. C., Bali, V., Narula, S., \& Kalra, M. (2014). Optimal component selection based on cohesion and coupling for component based software system under build-or-buy scheme. Journal of Computational Sciences, 5(2), $233-242$.

Jiang, Y., Song, Z., \& Kusiak, A. (2013). Very short-term wind speed forecasting with Bayesian structural break model. Energy Conversion and Management, 50(C), 637-647.

Kavasseri, R. G., \& Seetharaman, K. (2009). Day-ahead wind speed forecasting using f-ARIMA mpdels. Renewable Energy, 34(5), 1388-1393. doi:10.1016/j.renene.2008.09.006

Kumar, A., Jain, R., Pandey, A., Gupta, S. P., \& Garg, S. (2018). Electricity Load Forecasting Methods-A Review. International Journal of Research in Electronics and Computer Engineering, 6(3), 374-378.

Kumar, N., Mishra, B., \& Bali, V. (2018). A novel approach for blastit-induced fly rock predication based on particle swarm optimization and artificial neural network. In Proceedings of International Conference on Recent Advancement in Computers and Communication (pp. 19-27). Academic Press. doi:10.1007/978-981-10-8198-9_3

Lawan, S. M., Abidin, W. A. W. Z., Chai, W. Y., Baharun, A., \& Masri, T. (2014). Different Models of Wind Speed Prediction: A Comprehensive Review. International Journal of Scientific \& Engineering Research, 5(1), 1760-1768. 
Li, G., Shi, J., \& Zhou, J. (2011). Bayesian adaptive combination of short -term wind speed forecasts from neural network models. Renewable Energy, 36(1), 352-359.

Liu, D., Niu, D., Wang, H., \& Fan, L. (2014). Short-term wind speed forecasting using wavelet transform and support vector machines optimized by genetic algorithm. Renewable Energy, 62(1), 592-597.

Liu, H., Xiwie, M., \& Yanfei, L. (2018). Smart deep learning based wind speed prediction model using wavelet packet decomposition, convolution neural network and convolutional long short term memory network. Energy Conversion and Management, 166(1), 120-131.

Mishra, S. P., \& Dash, P. K. (2017). Short term wind speed prediction using multiple kernel pseudo inverse neural networks. International Journal of Automation and Computing, 15(1), 66-83. doi:10.1007/s11633-017-1086-7

Pourmousavi Kani, S. A., \& Ardehali, M. M. (2013). Very short-term wind speed prediction: A new artificial neural network-Markov chain model. Energy Conversion and Management, 52(1), 738-745. doi:10.1016/j. enconman.2010.07.053

Tronsco, A., Salcedo-sanz, S., Casanova-Mateo, C., Riquelmw, J. C., \& Prieto, L. (2015). Local models-based regression trees for very short-term wind speed predictions. Renewable Energy, 81(C), 589-598.

Wang, J., Qin, S., Zhou, Q., \& Jiang, H. (2015). Medium - term wind speeds forecasting utilizing hybrid models form three different sites in Xinjiang. Renewable Energy, 76(1), 91-101. doi:10.1016/j.renene.2014.11.011

Wang, J., \& Wang, Y. (2017). A novel wind speed forecasting model for wind farms of Northwest China. International Journal of Green Energy, 14(5), 463-478. doi:10.1080/15435075.2016.1278373

Yesilbudak, M., Sagiroglu, S., \& Colak, I. (2013). A new approach to very short term wind speed prediction using k-nearest neighbour classification. Energy Conversion and Management, 69(1), 77-86. doi:10.1016/j. enconman.2013.01.033

Yue, Y., Zhao, Y., Zhao, H., \& Wang, H. (2017). Short-term Wind Speed Combined Prediction for Wind Farms. In Proceedings of the International Conference on Mechatronics and Automation (pp. 6-9). Academic Press. doi:10.1109/ICMA.2017.8015781

Zhang, W., Wang, J., Wang, J., Zhao, Z., \& Tian, M. (2013). Short-term wind speed forecasting based on a hybrid mode. Applied Soft Computing, 13(1), 3225-3233. doi:10.1016/j.asoc.2013.02.016

Zhang, Y., Chen, B., Zhao, Y., \& Pan, G. (2018). Wind Speed Prediction of IPSO-BP Neural Network Based on Lorenz Disturbance. IEEE Access: Practical Innovations, Open Solutions, 6(1), 1-9.

Zhang, Y., Pan, G., Zhang, C., \& Zhao, Y. (2019). Wind speed prediction research with EMD-BP based on Lorenz disturbance. Electrical Engineering, 70(3), 198-207. doi:10.2478/jee-2019-0028

Zhao, X., Wei, H., Zhang, C., \& Zhang, K. (2017). Selective Sampling Using Active Learning for Short-term Wind Speed Prediction. In Proceedings of the 29th Chinese Control and Decision Conference (pp. 6717-6720). Academic Press. doi:10.1109/CCDC.2017.7978387 
Vikram Bali received his B. Tech (CSE) from REC, Kurukshetra, M.E. (CSE) from NITTTR, Chandigarh and PhD from Banasthali Vidyapith, Rajasthan. He has more than 18 years of rich academic experience. He is a Professor \& Head of Department (CSE) at JSS Academy of Technical Education, Noida. He is lifetime member of Indian Society for Technical Education (ISTE), Computer Society of India (CSI) and Institution of Engineers (IE). He has contributed 21 research papers in international journals and 7 research papers in national conferences/proceedings and edited books. He has also attended faculty enablement programme organised by Infosys and NASSCOM. He has been the member of board of studies of different Indian universities and a member of the organizing committee for various national and international seminar/conferences. He has written books on the fundamentals of cyber security and laws, software engineering, and operating systems. He is reviewer to many international journals of repute for publishers like Inderscience and IGI Global. His research interest includes software engineering, cyber security, automata theory, CBSS, and ERP.

Ajay Kumar is an Assistant Professor in Department of Computer Science \& Engineering at JSS Academy of Technical Education, Noida. He is pursuing a Ph.D. from J.C. Bose University of Science \& Technology, YMCA. He received his M.Tech degree in Computer Engineering (Computer Networking) from YMCA UST, Faridabad, India. He received his B.Tech degree in Computer Engineering from U.I.E.T, Maharishi Dayanand University Rohtak, India. His areas of interests are machine learning, semantic web and web services and information retrieval system. $\mathrm{He}$ is the member of the International Association of Engineers (IAENG).

Satyam Gangwar, received his M.Tech (Computer Science and Engineering) from the JSS Academy of Technical Education Noida (U.P.), India. He did his B.Tech (Computer Science \& Information Technology) from Mahatma Jyotiba Phule Rohilkhand University, Bareilly, India. His areas of interests are machine learning and data science. 